\title{
GRAVITATIONAL ORIGIN OF QUARK MASSES IN AN EXTRA-DIMENSIONAL BRANE WORLD
}

\author{
DAVID DOOLING AND KYUNGSIK KANG \\ Department of Physics, Brown University, Providence RI 02912 USA \\ E-mail: dooling@het.brown.edu,kang@het.brown.edu
}

\begin{abstract}
Using the warped extra dimension geometry of the many-brane extension of the Randall-Sundrum solution, we find a natural explanation for the observed quark masses of the three Standard Model (SM) generations. Localizing massless SM matter generations on neighboring 3-branes in an extra dimensional world leads to phenomenologically acceptable effective four dimensional masses arising from the coupling of the fermion field with the background metric. Thus this geometry can simultaneously address the gauge and quark mass hierarchy problems.
\end{abstract}

\section{Introduction}

The Standard Model (SM) provides an elegant mechanism by which the massive intermediate vector bosons $W^{ \pm}$and $Z$ acquire mass while the photon and gluons remain massless. Postulating the Higgs field to transform as a singlet under $S U(3)_{c}$ and a doublet under $S U(2)$, the $W^{ \pm}$and $Z$ masses at tree level are given in terms of $g_{1}, g_{2}$ and only one dimensionful parameter $v \sim 246 \mathrm{GeV}$. The SM does not provide such an economical explanation for the observed fermion masses. After spontaneous symmetry breaking, the quark mass term in the lagrangian reads

$$
\mathcal{L}_{\text {mass }}=\frac{v}{\sqrt{2}}\left(\overline{u_{L i}} h_{i j}^{(u)} u_{R j}+\overline{d_{L i}} h_{i j}^{(d)} d_{R j}\right)+\text { h.c. }
$$

where the $h_{i j}$ are arbitrary $3 \times 3$ complex Yukawa coupling matrices. Some predictive mechanism for fermion mass generation is needed so as to place the understanding of fermion mass on a par with that of gauge boson mass; i.e., to find an overriding principle that predicts the values of the quark and lepton mass matrix elements to be what they are.

Another failing of the SM is that of explaining in a natural way the existence of a light fundamental Higgs mass scale in comparison to the Planck scale, the gauge hierarchy problem. Traditional proposed solutions have been technicolor and supersymmetry, while recently it has been suggested that large extra dimensions (LED) may result in a change in how gravity behaves at high energies and thus allow room for only one fundamental scale in physics, the $\mathrm{TeV}$ scale 1 . However, this suggestion in its simplest forms does not really address the gauge hierarchy problem, but rather transforms it into 
a problem of disparate length scales. One needs to explain why the extra dimensions are so large. Randall and Sundrum observed that if there are in fact extra dimensions, our world is necessarily confined to a four dimensional submanifold, a 3-brane $\mathrm{B}$. It is then apparent that any 3-branes living in extra dimensions must be taken into account when determining the metric. The imposition of four-dimensional Poincare invariance generically results in a non-facorizable geometry with an associated warp factor. Unlike in the LED scenarios, the gauge hierarchy problem is resolved not with the single fundamental scale being the $\mathrm{TeV}$ scale, but with the fundamental scale being the Planck scale. Particle physics scales of $1 \mathrm{TeV}$ are reproduced after taking into account the effect of the warp factor on the visible brane and canonically normalizing the Higgs field, so that a Higgs VEV $246 \mathrm{GeV}$ may result even if the fundamental $V_{0}$ is of the order of the Planck scale.

In this paper, we ask the question "can the gauge hierarchy problem and the quark mass hierarachy be simultaneously explained in a minimal extension of the Randall-Sundrum solution?". In a recent paper , we used the LykkenRandall scenario $t$ of one hidden, positive tension brane located at the origin in a non-compact extra dimension. Treating as probes the branes where the SM fields are localized, we found that a phenomenologically acceptable quark mass spectrum results, but that the mixing parameters were inconsistent with experiment. Taking this discrepency as a signal for a more rigorous treatment, we refine the previous calculation. Using the full metric as determined by all the branes, including the previously dubbed probe branes, we solve exactly for the fermion field profile in the extra compact dimension with the original $\frac{S_{1}}{\mathbf{Z}_{2}}$ orbifold symmetry. This new implementation of the same essential mechanism as in $\mathrm{Z}$ is much more restrictive, and yet we still find the quark mass spectrum can be successfully produced and the gauge hierarchy problem resolved.

The outline of this paper is as follows. In Section II, we review the manybrane extension of the Randall-Sundrum solution 9 and solve for the profile of a massless fundamental five-dimensional fermion field in this geometry, as outlined in Bajc and Gabadadze 8. In Section III, we present our mechanism of a gravitational origin of quark masses, made possible by the identification of different SM flavors with the peaked profile of one fundmental field around different branes. This idea of localizing different flavors at different locations in higher-dimensional geography has been exploited recently in 1 .6. Finally, we present an example justifying our claim that variants of the RS scenario are capable of successfully addressing both hierarchies mentioned above. We then draw our conclusions and briefly mention further directions currently under investigation.

proc: submitted to World Scientific on February 24, 2019 


\section{Many-brane Extension of RS}

In $\mathbf{9}$, Hatanaka et al. have generalized the orginal RS solution with the $\frac{S_{1}}{\mathbf{Z}_{2}}$ orbifold symmetry to the case of many branes. The general configuration considered is that of $N$ parallel 3 -branes in five spacetime dimensions such that the $i^{\text {th }} 3$-brane located at $\phi_{i}$ has tension $V_{i}(i=1,2, \ldots, N)$ and $0<\phi_{1}<$ $\phi_{2}<\ldots<\phi_{N}<2 \pi$. This more general configuration allows for the possibility of different inter-brane bulk cosomological constants, and so the entire action may be written as

$$
S=S_{\text {grav }}+\sum_{i=1}^{N} S_{i}
$$

where

$$
\begin{gathered}
S_{\text {grav }}=\int d^{4} x \int_{0}^{2 \pi} \sqrt{G}\left\{2 M^{3} R-\sum_{i=1}^{N} \lambda_{i}\left[\theta\left(\phi-\phi_{i}\right)-\theta\left(\phi-\phi_{i+1}\right)\right]\right\} \\
S_{i}=\int d^{4} x \sqrt{-g^{(i)}}\left\{\mathcal{L}_{i}-V_{i}\right\}
\end{gathered}
$$

and $\theta$ is the Heaviside step function. The resulting five dimensional Einstein equations are

$$
\begin{gathered}
\sqrt{G}\left(R_{M N}-\frac{1}{2} G_{M N} R\right)=-\frac{1}{4 M^{3}}\left[\sum_{i=1}^{N} \Lambda_{i}\left[\theta\left(\phi-\phi_{i}\right)-\theta\left(\phi-\phi_{i+1}\right)\right] \sqrt{G} G_{M N}\right. \\
\left.+\sum_{i=1}^{N} V_{i} \sqrt{-g^{(i)}} g_{\mu \nu}^{(i)} \delta_{M}^{\mu} \delta_{N}^{\nu} \delta\left(\phi-\phi_{i}\right)\right]
\end{gathered}
$$

Taking the same form for the metric ansätze as in the original RS scenario so as to preserve four dimensional Poincare invariance,

$$
d s^{2}=e^{-2 \sigma(\phi)} \eta_{\mu \nu} d x^{\mu} d x^{\nu}-r_{c}^{2} d \phi^{2}
$$

one finds the solution

$$
\begin{aligned}
\sigma(\phi)= & \left(\lambda_{1}-0\right)\left(\phi-\phi_{1}\right)+\left(\lambda_{2}-\lambda_{1}\right)\left(\phi-\phi_{2}\right) \theta\left(\phi-\phi_{2}\right) \\
& +\ldots+\left(\lambda_{N}-\lambda_{N-1}\right)\left(\phi-\phi_{N}\right) \theta\left(\phi-\phi_{N}\right)
\end{aligned}
$$


where $S^{1}$ periodicity $\left(\sigma(0)=\sigma\left(r_{c}\right)\right)$ requires

$$
\sum_{i=1}^{N} \lambda_{i}\left(\phi_{i+1}-\phi_{i}\right)=0
$$

and $\lambda_{i}= \pm \sqrt{\frac{-\Lambda_{i} r_{c}^{2}}{24 M^{3}}}, \frac{V_{i} r_{c}}{12 M^{3}}=\lambda_{i}-\lambda_{i-1}$ for $(i=1,2, \ldots, N)$ and $\lambda_{0}=\lambda_{N}$

Given this general metric, we now wish to couple a fundamental, massless five-dimensional fermion field to it and solve for its profile in the bulk. In 8 , Bajc and Gabadadze showed that massless fermions can be localized around a single negative tension brane, but that no normalizable solution exists in the case of a single positive tension brane when the extra dimension is noncompact. However, in the case of a $\frac{S^{1}}{\mathbf{Z}_{2}}$ symmetric extra dimension, both negative and positive tension branes may exist and the fermion profile in the bulk will have local maxima around the negative tension branes and local minima around the positive tension branes.

Switching notation so that the extra dimension is parametrized by $y$ $\left(0<y<y_{c}\right)$, we work with the configuration of twentyfour branes all with the same magnitude of tension, such that

$$
\sigma(y)=\frac{1}{12} \sqrt{\frac{-6 \Lambda}{M^{3}}} y \theta(y)+\frac{1}{12 M^{3}} \sum_{i=2}^{24} V_{i}\left(y=y_{i}\right) \theta\left(y-y_{i}\right)
$$

where $V_{i}=\mp V$ as $i$ is even or odd and

$$
y_{c}=\frac{V}{\left(12 M^{3}\left(\frac{V}{12 M^{3}}-\frac{1}{12} \sqrt{-\frac{6 \Lambda}{M^{3}}}\right)\right)} \sum_{i=2}^{24} \beta_{i} y_{i}
$$

where $\beta_{i}=\mp 1$ as $i$ is even or odd.

Given this metric, the properly normalized massless fermion field profile in the bulk is given by

$$
\psi=\frac{1}{\sqrt{n}} e^{2 \sigma(y)}
$$

where $n=\int_{0}^{y_{c}} d y e^{\sigma(y)}$. The effective Newton constant is given by

$$
\begin{gathered}
M_{p l}^{2}=M^{3} y_{c} \int_{0}^{y_{c}} d y e^{-2 \sigma(y)} \\
M_{p l}^{2}=M^{3} y_{c}\left[\left(\frac{1}{2 \lambda_{1}}-\frac{1}{2 \lambda_{N}}\right)+\sum_{i=1}^{N}\left(\frac{1}{2 \lambda_{i}}-\frac{1}{2 \lambda_{i+1}}\right) e^{-2 \sigma(y)}\right]
\end{gathered}
$$


and so is of the order $M_{p l}^{-2}$ for every brane. Hence the $y_{i}$ can be chosen so as to generate an acceptable quark mass spectrum without conflicting with the observed strength of gravity.

\section{Gravitational Origin of Quark Masses}

As in 1 , we may naturally define an effective four-dimensional pair of quark mass matrices as

$$
M_{i j}=\frac{1}{2} \int_{o}^{y_{c}} d y e^{-4 \sigma(y)}\left(\psi_{i L}\left(\partial_{y} \psi_{j R}\right)-\left(\partial_{y} \psi_{i L}\right) \psi_{j R}\right)
$$

Up and down quark sector mass matrices may then be evaluated once we adopt a particular brane number - SM field dictionary. Separating left and right-handed components, we may identify brane number and flavor as $(2,4,6,8,10,12,14,16,18,20,22,24) \rightarrow$ $\left(d_{R}, u_{R}, d_{L}, u_{L}, s_{R}, c_{R}, s_{L}, c_{L}, b_{R}, t_{R}, b_{L}, t_{L}\right)$. Different flavors are identified with different local maxima of the fermion field in the extra dimension.

We now show an example supporting our claim that this geometry allows for a simultaneous taming of the two mass hierarchy problems. Working in units of the Planck mass, we choose the fundamental parameters as follows: $M=1, \Lambda=-1$ and $V=5.51$. The position of the first brane is at the

origin of the $\frac{S^{1}}{\mathbf{Z}_{2}}$ obifold, $y_{1}=0$. We then place the remaining branes at equal coordinate intervals further out in the $y$ direction. In this example, we choose $y_{2}=49, y_{3}=56, \ldots, y_{24}=203$. No new hierarchy is introduced, as $y_{c}$ is caculated to be $\sim 227$ Planck lengths.

We show in Fig. (1) a plot of the function $\sigma(y)$ of Eq. (9). The original RS scenario would provide the same picture up to $y_{2}=49$, the position of the first negative tension brane. In this case, the presence of the additional branes of alternating positive and negative tensions of equal magnitude allows for the appearance of the several local maxima and minima of the function $\sigma(y)$. In Fig. (2) and Fig. (3), we plot the properly normalized fermion field profile in the bulk for this particular choice of parameters. We see that the peaks associated with the lighter flavors have a markedly higher profile than those associated with the heavier flavors. Within the context of our proposed mechanism for quark mass generation, this observation makes perfect sense. Because the fermion field profile goes like the inverse of the warp factor, wherever the fermion profile is large, the interaction with the background metric is dampened, and wherever the fermion profile is small, the interaction with the background metric is enhanced.

proc: submitted to World Scientific on February 24, 2019 


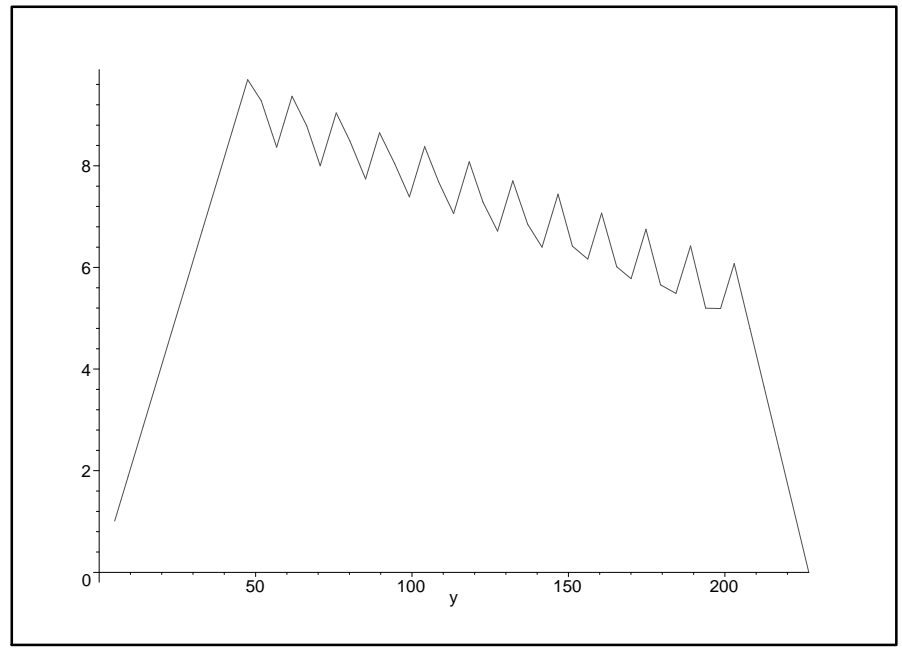

Fig. (1) The function $\sigma(y)$ plotted over the entire $\frac{S^{1}}{\mathcal{Z}_{2}}$ extra dimension.

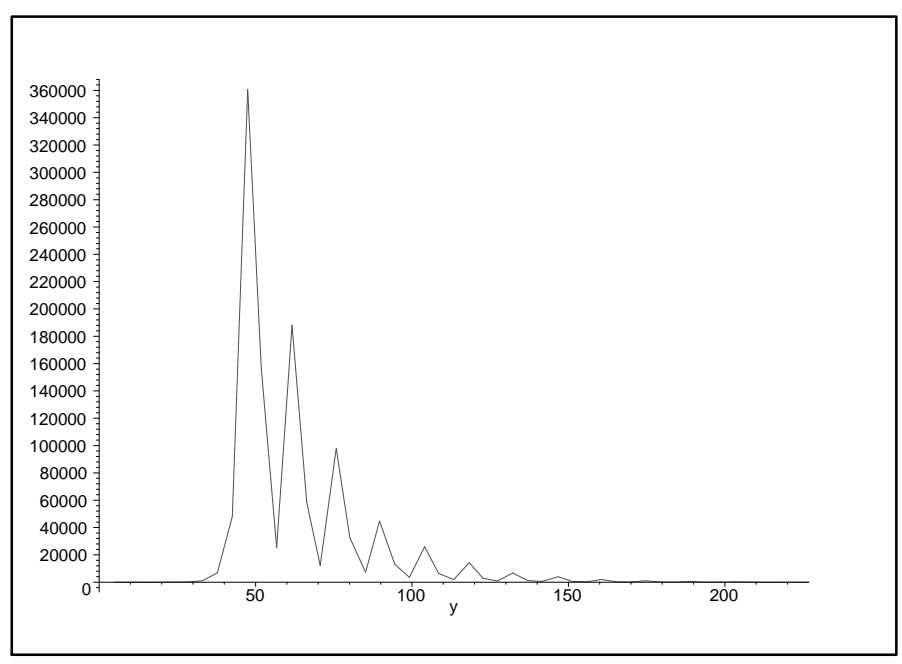

Fig. (2) The properly normalized fermion field profile plotted over the entire $\frac{S^{1}}{\mathcal{Z}_{2}}$ extra dimension. 


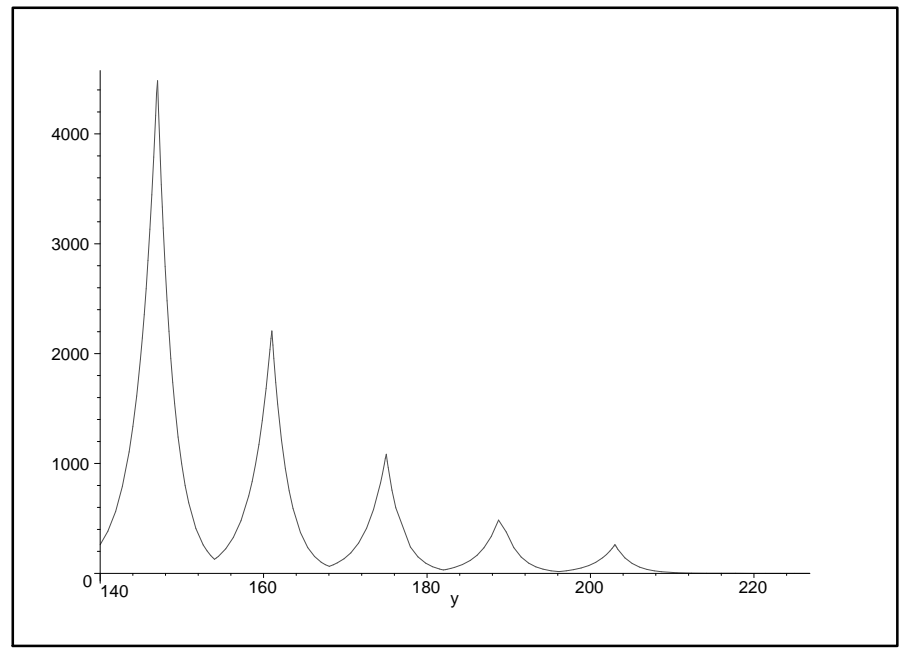

Fig. (3) The properly normalized fermion field profile plotted over the interval of the extra dimension around which the heavier flavors are peaked.

Using Eq. (14) and the above brane number - flavor dictionary, we can compute $\mathcal{M}_{u}=M_{u} M_{u}^{\dagger}$ and $\mathcal{M}_{d}=M_{d} M_{d}^{\dagger}$.

$$
\begin{gathered}
\mathcal{M}_{u}=\left(\begin{array}{ccc}
.177432 \times 10^{-40} & .249962 \times 10^{-38} & -.460803 \times 10^{-37} \\
.249962 \times 10^{-38} & .797256 \times 10^{-36} & -.137539 \times 10^{-34} \\
-.460803 \times 10^{-37} & -.137539 \times 10^{-34} & .238164 \times 10^{-33}
\end{array}\right) \\
\mathcal{M}_{d}=\left(\begin{array}{ccc}
.102262 \times 10^{-41} & .144233 \times 10^{-39} & -.265891 \times 10^{-38} \\
.144233 \times 10^{-39} & .460510 \times 10^{-37} & -.794454 \times 10^{-36} \\
-.265891 \times 10^{-38} & -.794454 \times 10^{-36} & .137567 \times 10^{-34}
\end{array}\right)
\end{gathered}
$$

where we remind the reader that we have been working in units of the Planck mass $M_{p l} \sim 1.221047 \times 10^{19} \mathrm{GeV}$. Computing the eigenvalues, one finds $m_{t} \sim$ $188 \mathrm{GeV}, m_{c} \sim .66 \mathrm{GeV}, m_{u} \sim .002 \mathrm{GeV}, m_{b} \sim 45 \mathrm{GeV}, m_{s} \sim .159 \mathrm{GeV}$ and $m_{d} \sim .0005 \mathrm{GeV}$. With the exception of the bottom quark, these values are in good agreement with observation and prompt us to take this model seriously. The mixing matrix calculated in the usual way from $V_{C K M}=U_{u}^{\dagger} U_{d}$ where $U_{u}^{\dagger} \mathcal{M}_{u} U_{u}=\operatorname{diag}\left(m_{u}^{2}, m_{c}^{2}, m_{t}^{2}\right)$ and $U_{d}^{\dagger} \mathcal{M}_{d} U_{d}=\operatorname{diag}\left(m_{d}^{2}, m_{s}^{2}, m_{b}^{2}\right)$ is computed to be essentially the $3 \times 3$ identity matrix. This result is in contrast to our previous implementation of this mechanism in $\mathrm{Z}$, in which there was too 
much mixing between the second and third generations. In our model, flavor mixing does not arise from the diagonalization of four dimensional quark mass matrices, but rather from wave function overlap, as in 0 .

As one may readily verify, varying the $y_{i}$ and the other fundamental parameters by factors of the order of 2 , or even relaxing the aestheticly pleasing requirements of equal magnitudes for the brane tensions and equal coordinate spacings for the brane positions in the extra dimension, one can indeed successfully produce a quark mass spectrum in all but perfect agreement with experimental observation. This variation may require some degree of moderate fine-tuning, but nothing like the fine-tuning associated with the gauge hierarchy problem within the context of the pure SM.

\section{Conclusions and Further Directions}

We have found a consistent, straightforward mechanism within the extra dimensional scenario to derive effective four dimensional quark mass matrices that are phenomenologically acceptable. Several issues warrant further investigation to either lend more credence to this model or to ban it to the bonfires of happy mathematical coincidences. The first issue is that of the stability of the brane coordinates, which enter strongly into the determination of the mass eigenvalues. Presumably, a simple generalization of the Goldberger-Wise mechanism could account for their stability. A more sophisticated explanation would not only account for their stability, but for the actual values needed to produce an acceptable mass spectrum. An additional point that needs to be addressed is our model's lack of CP violation. As is known, the SM mechanism for $\mathrm{CP}$ violation via a physically meaningful phase in the CKM matrix does not provide enough CP violation for the purposes of baryogenesis. Perhaps the source of $\mathrm{CP}$ violation and the origin of quark masses are different problems. To conclude, the RS scenario provides an intriguing resolution of the hierarchy problem. We find suggestive evidence that it may address the fermion mass hierarchy as well. Mass being the charge of spacetime-matter interactions, it seems only natural that the fermion mass hierarchy will be understood in terms of spacetime considerations as opposed to an internal flavor symmetry governing Yukawa-type interactions.

\section{Acknowledgements}

We wish to thank the Cosmo-2000 organizing committee for their examplary organization of this conference and their warm hospitality. DD also gratefully acknowledges the U.S. Dept. of Ed. for financial support via the Graduate

proc: submitted to World Scientific on February 24, 2019 
Assistance in Areas of National Need (GAANN) program and the NSF for support via a Dissertation Enhancement Award (INT-0083352). Support for this work was also provided in part by the U.S. Dept. of Energy grant DEFG02-91ER40688. Institutional report numbers for this work are BROWNHET-1239 and BROWN-TA-586.

\section{References}

1. I. Antoniadis, Phys. Lett. B 264 (1990) 377; I. Antoniadis, C. Munoz, M. Quiros, Nucl. Phys. B 397 (1993) 515; I. Antoniadis, N. Arkani-Hamed, S. Dimopoulos, G. Dvali, Phys. Lett. B 436 (1998) 257; K. R. Dienes, E. Dudas, T. Ghergetta, Phys. Lett. B 436 (1998) 55; Nucl. Phys. B 537 (1999) 47; N. Arkani-Hamed, S. Dimopoulos, G. Dvali, Phys. Lett. B 429 (1998) 263; H. Hatanaka, T. Inami, C. S. Lim, Mod. Phys. Lett. A 13 (1998) 2601; K. Yoshioka, Mod. Phys. Lett A 15 (2000) 29.

2. A. Perez-Lorenzana, hep-ph/0008333.

3. L. Randall, R. Sundrum, Phys. Rev. Lett. 83 (1999) 3370.

4. J. Lykken, L. Randall, JHEP 0006:014 (2000).

5. G. Dvali, M. Shifman, Phys. Lett. B 475 (2000) 295.

6. N. Arkani-Hamed, M. Schmaltz, Phys. Rev. D 61 (2000) 033005; T. Gherghetta, A. Pomarol, hep-ph/0003129; D. Kaplan, T. Tait, JHEP 0006:020 (2000).

7. D. Dooling, K. Kang, hep-ph/0006256.

8. B. Bajc, G. Gabadadze, Phys. Lett. B 474 (2000) 282.

9. H. Hatanaka, M. Sakawoto, M. Tachibana, K. Takenaga, Prog. Theor. Phys. 102 (1999) 1213. 